\title{
Efficacy and Safety of Immuno-based Therapy Versus Standard of Care in Extensive-stage Small- Cell Lung Cancer: An Updated Meta-analysis of Randomized Clinical Trials
}

Kun Liu

Xiangya Hospital Central South University

Youwen Zhu

Xiangya Hospital Central South University

Xue Zhou

Xiangya Hospital Central South University

Xiaolu Zhu

University of South China

Libo Peng

University of South China

Youyi Dai

Xiangya Hospital Central South University

Hong Zhu ( $\nabla$ zhuhong0719@126.com )

Xiangya Hospital Central South University

\section{Research Article}

Keywords: Extensive-stage Small-Cell Lung Cancer (ES-SCLC), immuno-based therapy, chemotherapy, survival, meta-analysis

Posted Date: January 17th, 2022

DOI: https://doi.org/10.21203/rs.3.rs-1200219/v1

License: (9) This work is licensed under a Creative Commons Attribution 4.0 International License. Read Full License 
Efficacy and Safety of Immuno-based Therapy Versus Standard of Care in Extensive-stage Small-Cell Lung Cancer : An Updated Meta-analysis of Randomized Clinical Trials

Running Head: Immuno-based Therapy for ES-SCLC.

Kun $\mathrm{Liu}^{1 *}$, Youwen $\mathrm{Zhu}^{1 *}$, Xue Zhou ${ }^{1}$, Xiaolu Zhu1, Libo Peng ${ }^{2}$, Youyi Dai ${ }^{1 \#}$, Hong $\mathrm{Zhu}^{1 \#}$

*contributed equally to this work

\# corresponding author

\section{Affiliations of authors:}

1. Department of Oncology, Xiangya Hospital, Central South University, Changsha, Hunan 410008, China

2. Department of Oncology, The Central Hospital of Loudi, the University of South China, Loudi, Hunan 417000, China

\section{\# Correspondence to:}

Hong Zhu:

First corresponding author

Department of Oncology, Xiangya Hospital, Central South University, Changsha, Hunan 410008, China

E-mail: zhuhong0719@126.com

Youyi Dai:

Second corresponding author

Department of Oncology, Xiangya Hospital, Central South University, Changsha, Hunan 410008, China

E-mail: 394744376@qq.com 


\section{ABSTRACT}

Introduction: Several clinical studies have shown the emergence of immune checkpoint inhibitors (ICIs) could break the standard of care and generate clinical benefits for Extensive-Stage Small-Cell Lung Cancer (ES-SCLC). We aim to assess the efficacy and safety of immuno-based therapy in patients with ES-SCLC.

Methods: Our search was performed in PubMed, Embase, Cochrane, and Web of Science databases for clinical studies published in English between Jan 1, 2000, and Nov 30, 2021. We extracted hazard ratios (HRs) and 95\% confidence intervals (CI) of overall survival (OS) and progression-free survival (PFS), objective response rate (ORR), grade 3 or higher treatment-related adverse events (AEs), and we calculated the $95 \%$ CI together with the incidence. In addition, subgroup analysis was performed for patients with brain metastasis, smoking, and liver metastasis.

Results: We identified 200 records, of which 6 studies (2,905 patients) were included. The combined results showed that immuno-based therapy significantly prolonged OS (HR,0.78; 95\%CI, 0.71 to 0.86 ; $\mathrm{p}<0.00001$ ), PFS (HR, 0.81,95\%CI, 0.75 to 0.87 ; $\mathrm{p}<0.00001)$ and ORR (HR, 1.06; 95\%CI, 1.00 to $1.11 ; \mathrm{p}=0.03)$. Serious AEs $(\geq$ 3 grade) were more frequent with immuno-based therapy [Risk Ratio (RR), 1.08; 95\%CI, 1.02 to $1.14 ; \mathrm{p}=0.007]$. Besides, the most common serious AEs were neutropenia $(25.1 \% ; 95 \% \mathrm{CI}, 18.9 \%$ to $32.6 \%)$, anemia $(12.2 \% ; 95 \% \mathrm{CI}, 9.6 \%$ to $15.4 \%)$ and thrombocytopenia $(8.3 \% ; 95 \% \mathrm{CI} 5.7 \%$ to $11.9 \%)$. Compared with standard of care, no obvious immuno-based therapy benefit was found for ES-SCLC patients with brain metastases at diagnosis $(\mathrm{HR}, 1.14 ; 0.87$ to $1.46 ; \mathrm{p}=0.32)$ and smoking (HR, $0.88,95 \% \mathrm{CI}, 0.73$ to $1.06 ; \mathrm{p}=0.19$ ).

Conclusion: Our study reveals that immuno-based therapy better improves the OS and PFS than standard chemotherapy in ES-SCLC patients. Hence, our results provide new insights into the efficacy and safety of ICIs combination strategies for guiding clinical practice.

Keywords: Extensive-stage Small-Cell Lung Cancer (ES-SCLC), immuno-based therapy, chemotherapy, survival, meta-analysis 


\section{Introduction}

Lung cancer ranks second in morbidity and first in mortality among all cancer types worldwide, with over $13 \%$ incidence and $22 \%$ mortality in $2021^{1}$. Small cell lung cancer (SCLC) comprises over 10\% of lung cancer, and over $60 \%$ were diagnosed with metastatic disease ${ }^{2,3}$. The worst prognosis is Extensive-Stage Small Cell Lung Cancer (ES-SCLC), with a 5-year survival rate of only $2 \%{ }^{4}$.

The emergence of immune checkpoint inhibitors (ICIs) has completely changed the treatment scheme of ES-SCLC, breaking the standard treatment of platinum-based chemotherapy in the past few decades. ICIs include anti-programmed cell death protein 1 (PD-1) and anti-PD-1 ligand antibody (PD-L1), against cytotoxic T lymphocyte antigen 4 (CTLA-4), which have been approved for ES-SCLC in the first-line setting (Atezolizumab, Durvalumab).

At present, several clinical trials have explored the potential clinical activity and toxicity profiles of ICIs-based therapy in ES-SCLC. For example, the IMpower133 study (a phase III, double-blind, randomized) demonstrated that adding atezolizumab (PD-L1) to chemotherapy for first-line treatment of ES-SCLC brought about significant improvement in overall survival (OS, hazard ratio [HR], 0.76; 95\% confidence interval $[\mathrm{CI}], 0.60$ to $0.95 ; \mathrm{P}=0.0154$ ) and progression-free survival (PFS, HR, $0.77 ; 95 \% \mathrm{CI}, 0.62$ to $0.96 ; \mathrm{P}=0.02$ ) versus chemotherapy ${ }^{5,6}$. The CASPIAN study (a phase III, open-label, sponsor-blind, randomized) showed that sustained OS benefit (HR $0.75 ; 95 \% \mathrm{CI}, 0.62$ to $0.91 ; \mathrm{P}=0.0032$ ) with the addition of durvalumab to chemotherapy in patients with ES-SCLC compared with a robust control. Nevertheless, the addition of durvalumab to tremelimumab plus chemotherapy did not significantly improve outcomes (OS, HR, $0 \cdot 82 ; 95 \% \mathrm{CI}, 0 \cdot 68$ to $1 \cdot 00 ; \mathrm{P}=0.045)^{7}$. The KEYNOTE-604 study (a phase III, double-blind, randomized) illustrated that pembrolizumab plus chemotherapy significantly improved PFS (HR, 0.75; 95\% CI, 0.61 to $0.91 ; \mathrm{P}=0.0023)$ and prolonged $\mathrm{OS}(\mathrm{HR}, 0.78 ; 95 \% \mathrm{CI}, 0.63$ to $0.97 ; \mathrm{P}=0.0164)$ compared with chemotherapy as first-line therapy for patients with ES-SCLC. ${ }^{8}$ The 
ECOG-ACRIN EA5161 study revealed that nivolumab plus chemotherapy improved the PFS (HR, 0.65; 95\% CI, 0.46 to $0.91 ; \mathrm{P}=0.012)$ and OS (HR, 0.67; 95\% CI, 0.46 to $0.98 ; \mathrm{P}=0.038$ ) compared to chemotherapy ${ }^{9}$. However, several studies have not achieved clinical benefits compared with chemotherapy in the treatment of ES-SCLC ${ }^{10,11}$. These studies revealed that the immunotherapy-based adverse events (AEs) were relatively high.

Therefore, different conclusions have been drawn regarding the immunotherapy-based efficacy and toxicity in patients with ES-SCLC. In this study, we conducted a meta-analysis of the efficacy and safety of ICIs-based combination therapy in patients with ES-SCLC. We aimed to summarize the combination regimen in phase II/III randomized clinical trials and report the clinical benefits and AEs of combination therapies. To provide a clinical reference, we also obtained the clinical benefits of several baseline populations and incidences of treatment-related AEs of the combined regimen.

\section{Method}

\subsection{Search strategy and study selection}

We prepared and wrote his meta-analysis according to the Preferred Reporting Items for Systematic Reviews and Meta-Analyses (PRISMA) statement ${ }^{12}$. We searched the PubMed, Cochrane, Embase, and Web of Science databases for articles published from January 1, 2000, to September 30, 2021. The key searching words were “extensive small-cell lung cancer, immunotherapy, PD-1, PD-L1, chemotherapy, and randomized clinical trial" (eTable 1 in supplementary materials). The inclusion criteria for literature were as follows: (1) patients diagnosed with advanced small cell lung cancer; (2) therapy comparing treatments that contain immunotherapy with chemotherapy alone; (3) phase II or phase III randomized controlled trials (RCT). And we excluded the reviews and systematic reviews, meta-analysis, and cost-effectiveness analysis. In addition, we also searched the abstracts from major conferences of the American Society of Clinical Oncology (ASCO) and 
the European Society of Medical Oncology (ESMO) meetings to find whether any additional studies have been published. The record was uploaded into EndNote (version 9.1) for further review.

\subsection{Data extraction}

A pre-determined information data table was prepared. Two authors extracted data independently from each retrieved literature or abstract and assessed the quality of the included article. When the two authors have different opinions, we decide whether to include the article in this study through consultation and discussion. We extracted the primary data, including authors, years of publication, treatment regimens of study and control groups, the total patients and events of each group, survival outcome data, and adverse events. We assessed the risk of bias of each RCT by the Cochrane Collaboration guidelines $^{13}$, which included the following aspect: selection bias, performance bias, detection bias, attrition bias, reporting bias, and other bias (eFigure 1 in supplementary materials). The primary outcomes were OS and PFS, and the secondary endpoints were ORR and AEs of grade greater than or equal to 3 .

\subsection{Statistical analysis}

We used HR as the effect sizes for OS and PFS, and the OR was used as the effect sizes for ORR and AEs. We used RevMan software (version 5.3), Stata software (version 14.0), R software (version4.1.1) to calculate the pooled HRs, ORRs, and AEs with $95 \%$ confidential intervals (CIs) with a random-effects model. We calculated the hazard ratio (HR) of OS and PFS, the relative risk (RR) of ORR, and the incidence of different types of adverse reactions, respectively. The corresponding 95\% confidence intervals were calculated. Subgroup analyses were performed on smoking characteristics, status with or without brain metastasis, status with or without liver metastasis. Heterogeneity was tested by $\mathrm{I}^{2}$ statistics and $\mathrm{X}^{2}$ test. If significant heterogeneity is obtained ( $\mathrm{p}$ $\leq 0.1$ or $\mathrm{I}^{2}<50 \%$ ), we use a random effect model; otherwise we use a 
fixed-effect model. For primary outcomes, publication bias was assessed by funnel plot asymmetry.

\section{Results}

\subsection{Characteristics of subjects in included trials}

A PRISMA flow diagram of the article searching process was illustrated in Figure 1. We found 200 records total, including 78 duplicate records, 49 articles of meta-analysis, systematic review, and cost-effectiveness analysis, 57 articles of a case report, non-randomized controlled trials and basic medical studies, and 10 ongoing clinical trials. Five of these clinical trials have published detailed results, and the remaining one was an abstract published on ASCO in 2020. The clinicopathological and therapeutical characteristics were summarized in table 1. In total, 1608 patients were treated with immune-based therapy, and 1297 patients were treated with chemotherapy alone.

\subsection{Analysis of the overall survival}

We performed this meta-analysis on eight treatment groups with immune-based therapy and six control groups with chemotherapy in six randomized controlled trials. From the results, we can see that the experimental group's curative effect, including immunotherapy, is better than that of the chemotherapy group (HR,0.78; 95\%CI, 0.71 to $0.86 ; \mathrm{p}<0.00001)$. The heterogeneity among the studies was no significant difference $\left(\mathrm{I}^{2}=0 ; \mathrm{P}<0.01\right)$ ( Figure $\left.2 \mathrm{~A}\right)$. At the same time, we analyzed the results according to whether the patients were smoking, accompanying brain metastasis, and accompanying liver metastasis. The patients were divided into two subgroups according to whether they smoked or not. There is a statistical significance between patients with brain metastasis and patients without brain metastasis. Analysis of brain metastasis or non-brain metastasis subgroups showed non-prolonged OS (HR, 1.14;0.87 to $1.46 ; \mathrm{p}=0.32)$ and prolonged $\mathrm{OS}(\mathrm{HR}, 0.82 ; 95 \% \mathrm{CI}, \quad 0.72$ to $0.95 ; \mathrm{P}=0.006)$, respectively( Figure 3). Besides, the results revealed that there was no benefit in 
the immune-based group between the smoking and no-smoking groups, with HR values of $0.88(95 \% \mathrm{CI}, 0.75$ to $1.03 ; \mathrm{p}=0.12)$ and $0.88(95 \% \mathrm{CI}, 0.73$ to 1.06 ; $\mathrm{p}=0.19)$ (eFigure 3 in supplementary materials). What's more, whether accompanying liver metastasis, patients both benefit from immune-based therapy, with HR value for liver metastasis group of 0.83 (95\%CI, 0.71 to 0.96 ; $\mathrm{P}=0.01)$ and $0.74(95 \% \mathrm{CI}, 0.65$ to $0.85 ; \mathrm{P}<0.0001)$ for no liver metastasis group (eFigure 4 in supplementary materials). We also performed the funnel plot for OS that revealed symmetry through visual observation (eFigure $2 \mathrm{~A}$ in supplementary materials).

\subsection{Evaluation of the progression-free survival}

Regarding PFS, immuno-based therapy showed a better PFS than chemotherapy alone (HR, 0.81,95\%CI, 0.75 to $0.87 ; \mathrm{p}<0.00001)$. As depicted in Figure 2B, nivolumab demonstrated possibly stronger effects than other immunotherapy, although there are fewer people in ECOG-ACRIN EA5161. There was no significant difference in heterogeneity among the studies. We also made the funnel plot for PFS that displayed symmetry through visual observation (eFigure 2B in supplementary materials).

\subsection{Assessment of the overall response rate}

We further explored the ORR among cohorts. In total, 1608 patients were assigned to the experimental group with immunotherapy from the six clinical trials, and 1297 patients were assigned to the chemotherapy alone group. The results demonstrated that the immunotherapy group hasn't shown statistical superiority over the chemotherapy group (HR, 1.06; 95\% CI, 1.00 to $1.11 ; \mathrm{p}=$ 0.03 ). Moreover, there was no significant difference in the heterogeneity among the studies $\left(\mathrm{I}^{2}=0.26, \mathrm{p}<0.01\right)$ ( Figure $\left.2 \mathrm{C}\right)$.

\subsection{Comparing of the toxicity}

Besides the efficacy, we also compared the treatment safety between groups. The results manifested that the incidence of AEs in the experimental group with 
immunotherapy was slightly higher than that in the chemotherapy group [Risk Ratio (RR), $1.08 ; 95 \% \mathrm{CI}, 1.02$ to $1.14 ; \mathrm{p}=0.007$ (Figure 2D). In addition, we also counted and summarized the incidence of adverse events. The results indicated that neutropenia had the highest incidence $(25.1 \% ; 95 \% \mathrm{CI}, 18.9 \%$ to $32.6 \%)$, followed by anemia $(12.2 \% ; 95 \% \mathrm{CI}, 9.6 \%$ to $15.4 \%)$ and thrombocytopenia $(8.3 \%$; $95 \% \mathrm{CI}, 5.7 \%$ to $11.9 \%)$ ( Figure 4$)$.

\section{Discussion}

With high metastasis potential and poor prognosis, the treatment of small cell lung cancer has been a difficult but attractive clinical problem. Over the past 30 years, platinum-containing chemotherapy has been the standard treatment ${ }^{14}$. We conducted this meta-analysis with the continuous development of immunotherapy drugs and the publication and the update of more and more relative clinical trial results ${ }^{15,16}$. Compared with previous studies ${ }^{17}{ }^{18}$, we have updated the data from the latest clinical trials, and our conclusions on safety are different from those of previous studies.

In the CASPIAN study ${ }^{7}$, the Durvalumab plus chemotherapy group showed the benefits of OS and PFS. The overall survival rate of 24 months in the durvalumab plus tremelimumab plus chemotherapy group was higher than that in the chemotherapy group. In addition, in the 12-month and 24-month progression-free survival rate, the progression-free survival rate of durvalumab plus tremelimumab plus chemotherapy group was higher than that of chemotherapy. However, there was no difference in median overall survival between the durvalumab plus tremelimumab plus chemotherapy and the chemotherapy alone groups. Moreover, adverse reactions (21.4\% vs. $10.2 \%$ vs. 9.4\%) were more frequent in the durvalumab plus tremelimumab plus chemotherapy group than the others $(21.4 \%$ vs. $10.2 \%$ vs. $9.4 \%)$. The higher incidence of adverse events in patients with durvalumab plus tremelimumab 
plus chemotherapy might finally lead to a lower object response and median overall survival. And in CheckMate 451, Nivolumab plus Ipilimumab, as maintenance, did not prolong OS for patients with ED-SCLC ${ }^{19}$. But in non-small cell lung cancer, double ICIs-based treatment showed more effectiveness than single ICIs-based treatment in squamous and PD-L1 $<1 \%$ subgroups ${ }^{20}$. However, in non-small cell lung cancer, double ICIs-based treatment showed superior effectiveness than single ICIs-based treatment in squamous and PD-L1 $<1 \%$ subgroups. ${ }^{20}$ Meanwhile, in clear cell renal cell carcinoma, nivolumab + ipilimumab showed higher OS $(\mathrm{HR}=0.69 ; 95 \% \mathrm{CI} 0.59$ to 0.81 ) and ORR (39.1\% vs. $32.4 \%$ ), compared to sunitinib. ${ }^{21}$ And nivolumab + ipilimumab showed a better effect in reducing the diameter of target renal tumors $^{22}$. Moreover, patients with melanoma benefited a lot from nivolumab plus ipilimumab, and its median OS was 72.1 months, while the median OS was 36.9 and 19.9 months in nivolumab and ipilimumab groups, respectively ${ }^{23,} 24$. Although PD-L1 expression was not an absolute prognostic biomarker, we thought the unexpected effect of double ICIs had a relationship with the lower expression of PD-L1 in small cell lung cancer. Therefore, there is a question that we may need to consider in the process of clinical treatment, whether the combination of two immunotherapy drugs can bring considerable benefits to patients under acceptable economic conditions in small cell lung cancer.

However, what's exciting is the result of the ECOG-ACRINEA5161 study released by ASCO in 2020, showing that the nivolumab plus chemotherapy group could benefit both OS and PFS in the chemotherapy alone group ${ }^{9}$. At the same time, the IMpower133 test updated the OS results. In the case of equal safety, the median follow-up time of the atezolizumab plus chemotherapy group was prolonged to 22.9 months, which showed significant benefits compared with the chemotherapy group 5 . 
Therefore, we conducted a meta-analysis of the efficacy and safety of immuno-based therapy versus chemotherapy alone. The final results showed that the treatment regimen containing immunotherapy had better benefits than chemotherapy alone, both for OS (HR, 0.78; 95\%CI, 0.71 to 0.86$)$ and PFS (HR, $0.81 ; 95 \% \mathrm{CI}, 0.75$ to 0.87$)$. The subgroup analysis revealed that the regimen containing immunotherapy did not prolong the OS of patients with brain metastasis. In the Keynote-604 study, more patients had baseline BMS than in the IMpower133 and CASPIAN studies (14.5\% vs. $8.5 \%$ vs. $10 \%)$, and there was a baseline BMS imbalance $(14.5 \%$ vs. 9.8\%) between the pembrolizumab and the control group, which may be part of the reason for the failure of the Keynote-604 study ${ }^{6-8}$. The regimen including immunotherapy could increase OS compared with the chemotherapy group with or without liver metastasis. Unfortunately, considering the lack of sufficient data in included studies, we could not analyze whether the status of PD-L1 or tumor mutation burden (TMB) affected the prognosis of tumor and prediction of therapeutic effect. And conventionally, we regarded them as predictive biomarkers. Patients with higher TMB had a longer PFS or OS ${ }^{25-28}$. In small cell lung cancer, the estimate of PD-L1 expression was 26\% (95\%CI, 17.0\%-37\% $)^{29}$, not expected high like non-small cell lung cancer. And Tsakonas $G$ found that Brain metastasis samples, compared to primary tumor samples, downregulate genes related to immune response and immune cell activation in non-small cell lung cancer ${ }^{30}$. This unique gene downregulation pattern and low expression of PD-L1 may have a function in small cell lung cancer that causes a poorer benefit in the brain metastasis subgroup.

In addition, we also compared the adverse reactions. The results were also the same as we initially expected but differed from the previous meta-analysis, the incidence of adverse reactions in the immunotherapy group was slightly higher than that in the chemotherapy group. Subsequently, we performed a 
meta-analysis on the occurrence probability of various adverse reactions in the immunotherapy group, and the results showed that the incidence of hematological toxicity such as neutropenia and anemia was high, which may be related to combined chemotherapy. Myelosuppression is the most common chemotherapeutic adverse event, especially neutropenia. Chemotherapy agents can inhibit the function or proliferation of immune cells. For instance, vinblastine can destroy the microtubule protein to induce apoptosis in dividing cells. $^{31}$ Furthermore, immune-related adverse events (irAEs) are generally caused by immune cells' abnormal function, like B cell generating Pathogenic autoantibodies. So the common irAEs are ICIs-induced pneumonia, type 1 diabetes, and hypothyroidism. And the incidence of irAEs such as pneumonia and rash is relatively low and suggested that the incidence of adverse reactions of immunotherapy is lower than that of chemotherapy. This result is consistent with the newest systematic review of the treatment-related adverse events of PD-1 and PD-L1 ${ }^{32}$. However, suppose we want to make an accurate comparison, we still need to know specifically about the incidence of immune-related adverse reactions in the study and make a statistical analysis.

There are also some limits. We did not distinguish between studies in terms of chemotherapy regimens but instead treated them as a whole, which overlooked a part of the heterogeneity of study designs. Due to the early start of the clinical trial, paclitaxel and carboplatin were used in the chemotherapy regimen of the ipilimumab trial in 2013, which increased the heterogeneity between studies. In several clinical trials included, most of the treatment regimens in the trial group were single ICIs combined with chemotherapy, but there is a treatment regimen that contains double ICIs. Then, as the EP regimen is more effective, the rest of the clinical trials mostly use etoposide plus platinum chemotherapy control. This is also an essential source of heterogeneity among studies. 


\section{Conclusion}

In conclusion, this meta-analysis of randomized clinical trials performed to evaluate the efficacy and safety of immuno-based therapy in ES-SCLC patients suggests that ICIs combination therapy significantly improves the OS and PFS than standard chemotherapy. It also indicates that the incidence of AEs of this regimen is higher, but it was well tolerated. Hence, our results provide new insights into the efficacy and safety of PD-1 or PD-L1 inhibitor-based combination therapies for guiding clinical practice.

\section{Abbreviations}

SCLC: Small cell lung cancer; ES-SCLC: extensive-stage small cell lung cancer; ICIs: Immune checkpoint inhibitors; PD-1: Programmed cell death 1; PD-L1: Programmed cell death 1 ligand 1; CTLA-4: Cytotoxic T lymphocyte-associated antigen 4; OS: Overall survival; PFS: Progression-free survival; HR: Hazard ratio; ORR: objective response rate; AEs: adverse events; PRISMA: the Preferred Reporting Items for Systematic Reviews and Meta-Analyses; RCT: Randomized controlled trials; ASCO: the American Society of Clinical Oncology; ESMO: the European Society of Medical Oncology; CI: Confidential intervals; RR: Relative risk; TMB: Tumor mutation burden; irAEs: Immune-related adverse events;

\section{Acknowledgments}

All authors had full access to all of the data in this study and take complete responsibility for the integrity of the data and accuracy of the data analysis.

\section{Author' contributions}

K.L., Y.W.Z., X.Z, X.L.Z, L.B.P, Y.Y.D, H.Z. Designed experiment: K.L., Y.W.Z., Y.Y.D, and H.Z. Performed the experiments: K.L., Y.W.Z., X.Z, and X.L.Z. Analyzed the data: K.L., Y.W.Z., and L.B.P. Wrote the manuscript: K.L., 
Y.W.Z., X.Z. K.L. and Y.W.Z. contributed equally to this article. H.Z. and Y.Y.D co-corresponding author. All authors have read and approved the manuscript.

\section{Funding}

Not applicable.

\section{Availability of data and materials}

All authors had full access to all of the data in this study and take complete responsibility for the integrity of the data and accuracy of the data analysis. The datasets generated and/or analyzed during the current study are available from the corresponding author on reasonable request.

\section{Conflicts of interest}

All of the authors have indicated that they have no competing interests in the content of the article. This manuscript is original and has not been previously published, nor has it been simultaneously submitted to any other journal.

\section{Ethics approval and consent to participate}

Not applicable.

\section{Competing interests}

The authors declare that they have no competing interests. 


\section{Reference}

1 Siegel RL, Miller KD, Fuchs HE et al. Cancer Statistics, 2021. CA Cancer J Clin 2021; 71 (1): 7-33.

2 Nicholson AG, Chansky K, Crowley J et al. The International Association for the Study of Lung Cancer Lung Cancer Staging Project: Proposals for the Revision of the Clinical and Pathologic Staging of Small Cell Lung Cancer in the Forthcoming Eighth Edition of the TNM Classification for Lung Cancer. J Thorac Oncol 2016; 11 (3): 300-311.

3 Rudin CM, Giaccone G, Ismaila N. Treatment of Small-Cell Lung Cancer: American Society of Clinical Oncology Endorsement of the American College of Chest Physicians Guideline. J Oncol Pract 2016; 12 (1): 83-86.

4 Zimmerman S, Das A, Wang S et al. 2017-2018 Scientific Advances in Thoracic Oncology: Small Cell Lung Cancer. J Thorac Oncol 2019; 14 (5): 768-783.

5 Liu SV, Reck M, Mansfield AS et al. Updated Overall Survival and PD-L1 Subgroup Analysis of Patients With Extensive-Stage Small-Cell Lung Cancer Treated With Atezolizumab, Carboplatin, and Etoposide (IMpower133). Journal of clinical oncology : official journal of the American Society of Clinical Oncology 2021; 39 (6): 619-630.

Horn L, Mansfield AS, Szczęsna A et al. First-Line Atezolizumab plus Chemotherapy in Extensive-Stage Small-Cell Lung Cancer. The New England journal of medicine 2018; 379 (23): 2220-2229.

7 Goldman JW, Dvorkin M, Chen Y et al. Durvalumab, with or without tremelimumab, plus platinum-etoposide versus platinum-etoposide alone in first-line treatment of extensive-stage small-cell lung cancer (CASPIAN): updated results from a randomised, controlled, open-label, phase 3 trial. The Lancet Oncology 2021; 22 (1): 51-65.

8 Rudin CM, Awad MM, Navarro A et al. Pembrolizumab or Placebo Plus Etoposide and Platinum as First-Line Therapy for Extensive-Stage Small-Cell Lung Cancer: Randomized, Double-Blind, Phase III KEYNOTE-604 Study. Journal of clinical oncology : official journal of the American Society of Clinical Oncology 2020; 38 (21): 2369-2379.

9 Leal T, Wang YT, Dowlati A et al. Randomized phase II clinical trial of cisplatin/carboplatin and etoposide (CE) alone or in combination with nivolumab as frontline therapy for extensive-stage small cell lung cancer (ES-SCLC): ECOG-ACRIN EA5161. Journal of Clinical Oncology 2020; 38 (15).

10 Reck $\mathrm{M}$, Bondarenko I, Luft $\mathrm{A}$ et al. Ipilimumab in combination with paclitaxel and carboplatin as first-line therapy in extensive-disease-small-cell lung cancer: results from a randomized, double-blind, multicenter phase 2 trial. Annals of oncology : official journal of the European Society for Medical Oncology 2013; 24 (1): 75-83.

11 Reck M, Luft A, Szczesna A et al. Phase III Randomized Trial of Ipilimumab Plus Etoposide and Platinum Versus Placebo Plus Etoposide and Platinum in Extensive-Stage Small-Cell Lung Cancer. Journal of clinical oncology 2016; 34 (31): 3740-3748.

12 Moher D, Liberati A, Tetzlaff J et al. Moher D, Liberati A, Tetzlaff J, Altman DG, Group PPreferred reporting items for systematic reviews and meta-analyses: the PRISMA statement. PLoS Med 6: e1000097. Open Medicine 2009; 3 (3): e123-130. Higgins JP, Altman DG. Assessing Risk of Bias in Included Studies: Cochrane Handbook for Systematic Reviews of Interventions.

14 Farago AF, Keane FK. Current standards for clinical management of small cell lung cancer. 
Translational lung cancer research 2018; 7 (1): 69-79.

Rijavec E, Genova C, Biello F et al. Current state of the art and future perspectives with immunotherapy in the management of small cell lung cancer. Expert review of respiratory medicine 2021; 15 (11): 1427-1435.

Ortega-Franco A, Ackermann C, Paz-Ares L et al. First-line immune checkpoint inhibitors for extensive stage small-cell lung cancer: clinical developments and future directions. ESMO open 2021; 6 (1): 100003.

17 Wu J, Zhang A, Li L et al. Meta-analysis of the Efficacy and Tolerability of Immune Checkpoint Inhibitors Combined With Chemotherapy in First-line Treatment of Small Cell Lung Cancer. Clin Ther 2021; 43 (3): 582-593.e582.

18 Gristina V, Galvano A, Castellana L et al. Is there any room for PD-1 inhibitors in combination with platinum-based chemotherapy as frontline treatment of extensive-stage small cell lung cancer? A systematic review and meta-analysis with indirect comparisons among subgroups and landmark survival analyses. Therapeutic advances in medical oncology 2021; 13: 17588359211018018.

19 Owonikoko TK, Park K, Govindan R et al. Nivolumab and Ipilimumab as Maintenance Therapy in Extensive-Disease Small-Cell Lung Cancer: CheckMate 451. Journal of clinical oncology : official journal of the American Society of Clinical Oncology 2021; 39 (12): 1349-1359.

20 Xu Q, Zhang X, Huang M et al. Comparison of Efficacy and Safety of Single and Double Immune Checkpoint Inhibitor-Based First-Line Treatments for Advanced Driver-Gene Wild-Type Non-Small Cell Lung Cancer: A Systematic Review and Network Meta-Analysis. Front Immunol 2021; 12: 731546.

21 Regan MM, Jegede OA, Mantia CM et al. Treatment-free Survival after Immune Checkpoint Inhibitor Therapy versus Targeted Therapy for Advanced Renal Cell Carcinoma: 42-Month Results of the CheckMate 214 Trial. Clinical cancer research : an official journal of the American Association for Cancer Research 2021.

22 Albiges L, Tannir NM, Burotto $M$ et al. First-line Nivolumab plus Ipilimumab Versus Sunitinib in Patients Without Nephrectomy and With an Evaluable Primary Renal Tumor in the CheckMate 214 Trial. European urology 2021.

23 Larkin J, Chiarion-Sileni V, Gonzalez R et al. Five-Year Survival with Combined Nivolumab and Ipilimumab in Advanced Melanoma. The New England journal of medicine 2019; 381 (16): 1535-1546.

24 Wolchok JD, Chiarion-Sileni V, Gonzalez R et al. Long-Term Outcomes With Nivolumab Plus Ipilimumab or Nivolumab Alone Versus Ipilimumab in Patients With Advanced Melanoma. Journal of clinical oncology : official journal of the American Society of Clinical Oncology 2021: Jco2102229.

25 Gandara DR, Paul SM, Kowanetz M et al. Blood-based tumor mutational burden as a predictor of clinical benefit in non-small-cell lung cancer patients treated with atezolizumab. Nature medicine 2018; 24 (9): 1441-1448.

26 Paz-Ares LG, Ramalingam SS, Ciuleanu TE et al. First-Line Nivolumab Plus Ipilimumab in Advanced NSCLC: 4-Year Outcomes From the Randomized, Open-Label, Phase 3 CheckMate 227 Part 1 Trial. Journal of thoracic oncology : official publication of the International Association for the Study of Lung Cancer 2021. 
Reck M, Rodríguez-Abreu D, Robinson $A G$ et al. Five-Year Outcomes With Pembrolizumab Versus Chemotherapy for Metastatic Non-Small-Cell Lung Cancer With PD-L1 Tumor Proportion Score $\geqslant 50$. Journal of clinical oncology : official journal of the American Society of Clinical Oncology 2021; 39 (21): 2339-2349.

28 Reck M, Rodríguez-Abreu D, Robinson AG et al. Updated Analysis of KEYNOTE-024: Pembrolizumab Versus Platinum-Based Chemotherapy for Advanced Non-Small-Cell Lung Cancer With PD-L1 Tumor Proportion Score of 50\% or Greater. Journal of clinical oncology : official journal of the American Society of Clinical Oncology 2019; 37 (7): 537-546.

29 Acheampong E, Abed A, Morici M et al. Tumour PD-L1 Expression in Small-Cell Lung Cancer: A Systematic Review and Meta-Analysis. Cells 2020; 9 (11).

30 Tsakonas G, Lewensohn R, Botling J et al. An immune gene expression signature distinguishes central nervous system metastases from primary tumours in non-small-cell lung cancer. European journal of cancer (Oxford, England : 1990) 2020; 132: 24-34.

31 Shurin MR, Naiditch $\mathrm{H}$, Gutkin DW et al. ChemolmmunoModulation: immune regulation by the antineoplastic chemotherapeutic agents. Curr Med Chem 2012; 19 (12): 1792-1803.

32 Zhou X, Yao Z, Bai $\mathrm{H}$ et al. Treatment-related adverse events of PD-1 and PD-L1 inhibitor-based combination therapies in clinical trials: a systematic review and meta-analysis. The Lancet Oncology 2021; 22 (9): 1265-1274. 


\section{FIGURE LEGENDS}

Figure 1. Flow chart of study selection.

Figure 2. Meta-analysis of immunotherapy-based therapies vs chemotherapy alone. (A) Overall survival, (B) progression-free survival, (C) objective response rate, and (D) $\geq$ grade 3 adverse events.

Figure 3. Meta-analysis of immunotherapy-based therapies vs chemotherapy alone. (A) Overall survival in patients with brain metastases, and (B) overall survival in patients with non-brain metastases.

Figure 4. Rank forest plot of overall incidences of treatment-related adverse events in immunotherapy-based therapies.

Table 1. Characteristics of RCTs included in the meta-analysis.

eFigure 1. Risk of bias of evidence assessment.

eFigure 2. The funnel plots for overall survival (A) and progression-free survival (B).

eFigure 3. Meta-analysis of immunotherapy-based therapies vs chemotherapy alone. (A) Overall survival in smoker, and (B) overall survival in non-smoker.

eFigure 4. Meta-analysis of immunotherapy-based therapies vs chemotherapy alone. (A) Overall survival in patients with liver metastases, and (B) Overall survival in patients with non-liver metastases.

eTable 1. Search strategy. 
Figures
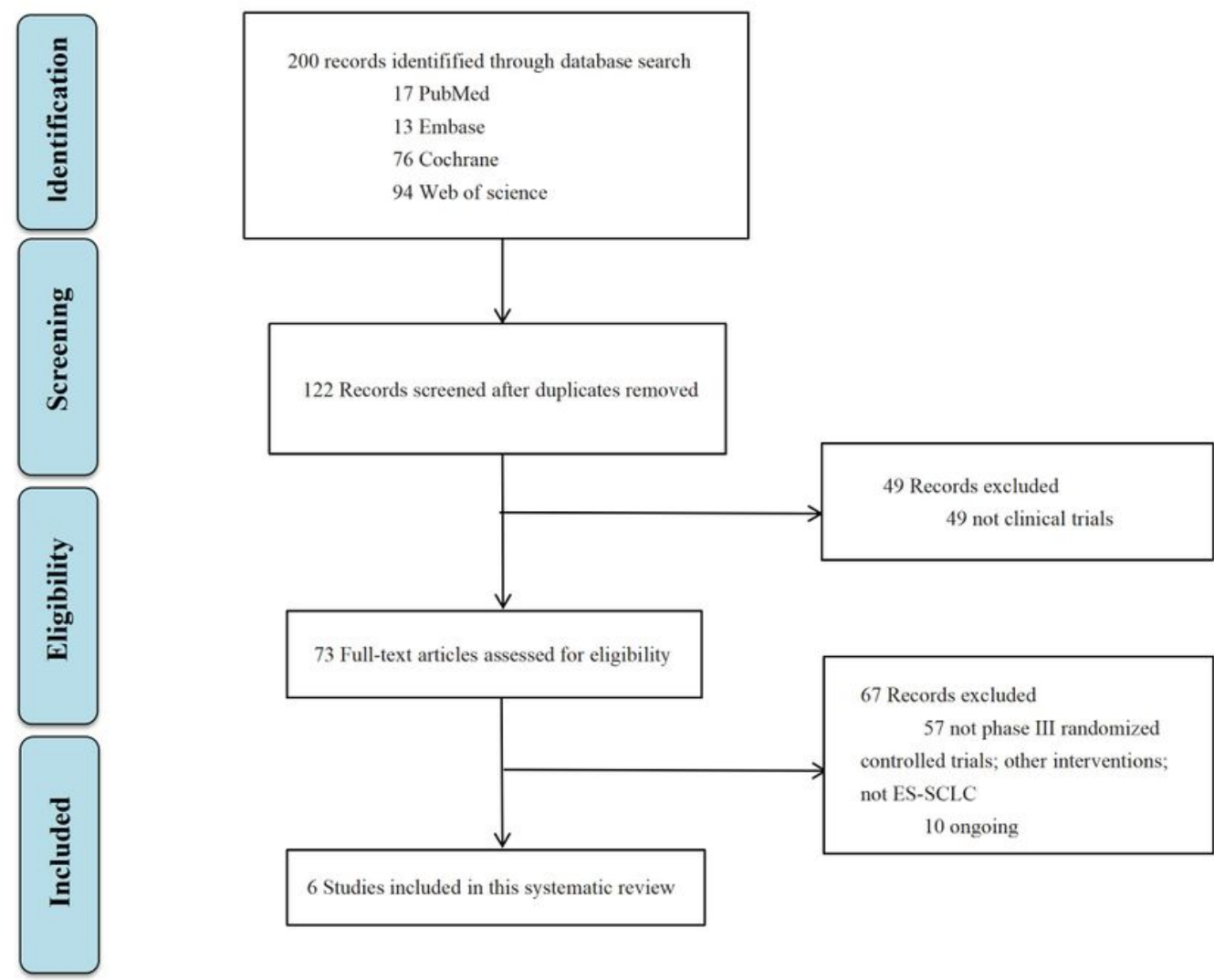

Figure 1

Flow chart of study selection. 


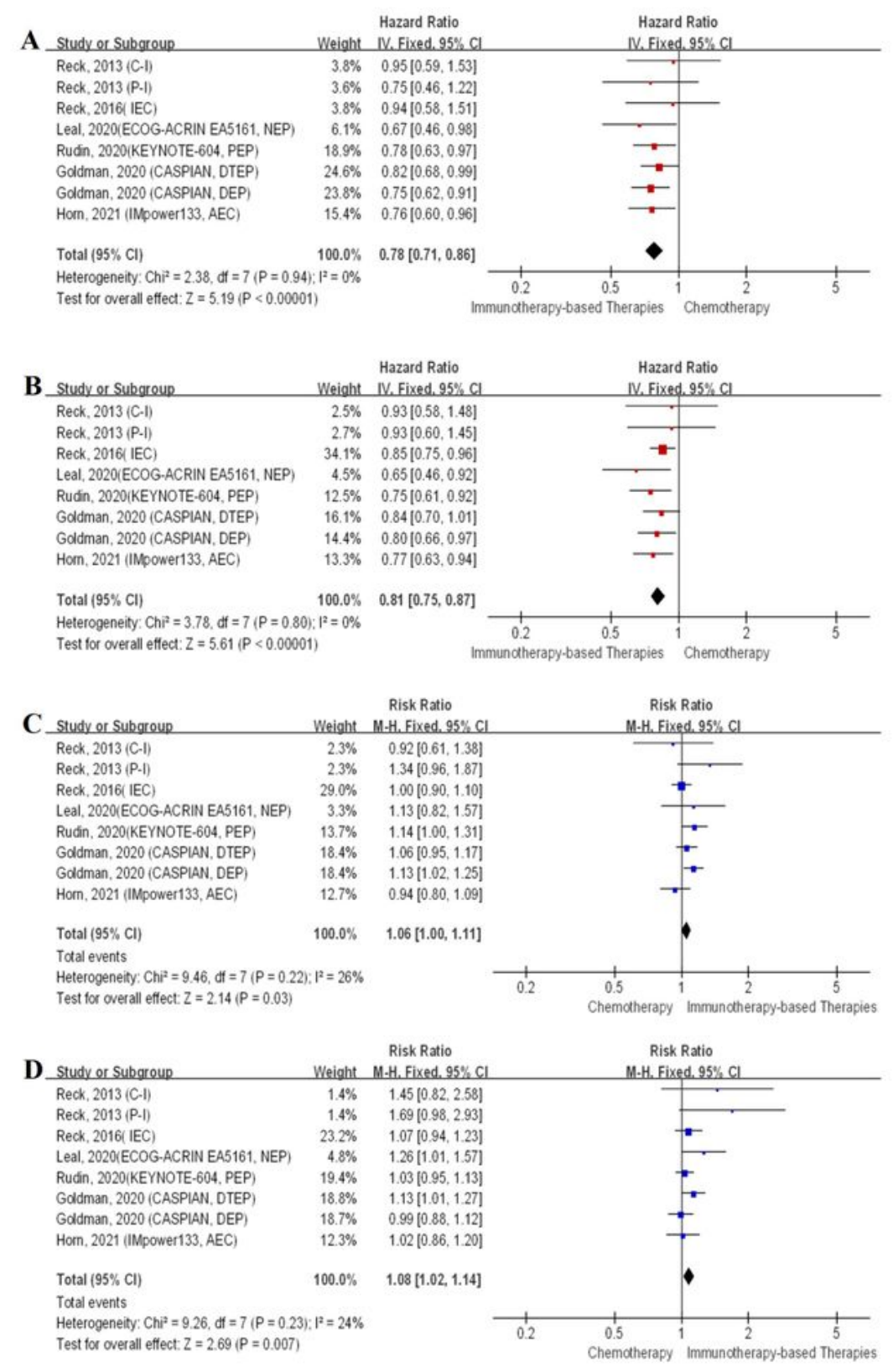

Figure 2

Meta-analysis of immunotherapy-based therapies vs chemotherapy alone. (A) Overall survival, (B) progression-free survival, (C) objective response rate, and (D) $\geq$ grade 3 adverse events. 


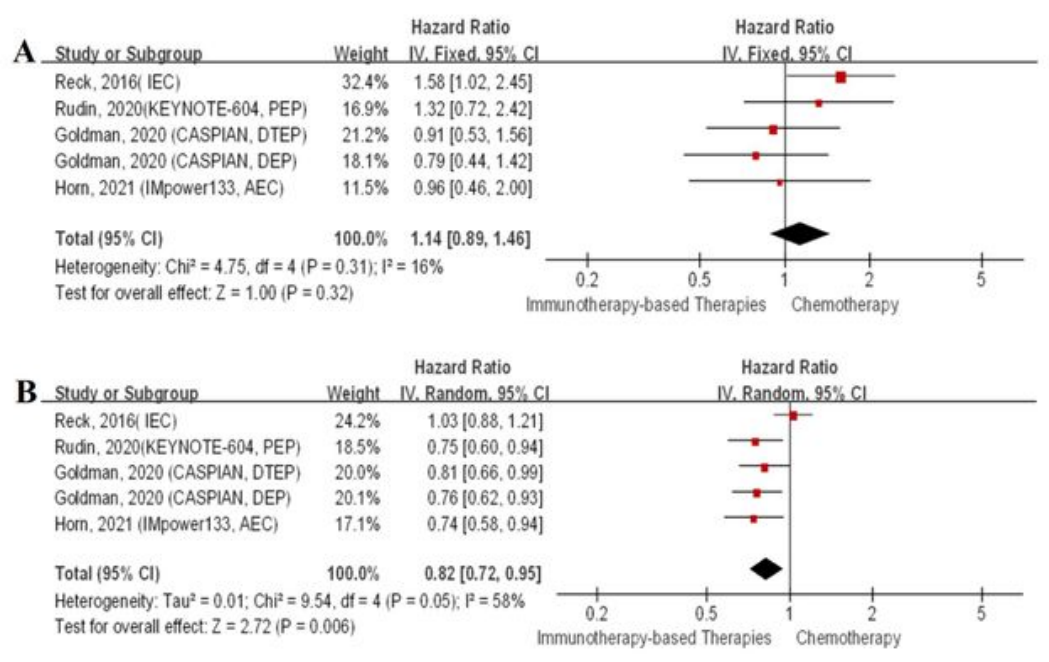

\section{Figure 3}

Meta-analysis of immunotherapy-based therapies vs chemotherapy alone. (A) Overall survival in patients with brain metastases, and (B) overall survival in patients with non-brain metastases. 


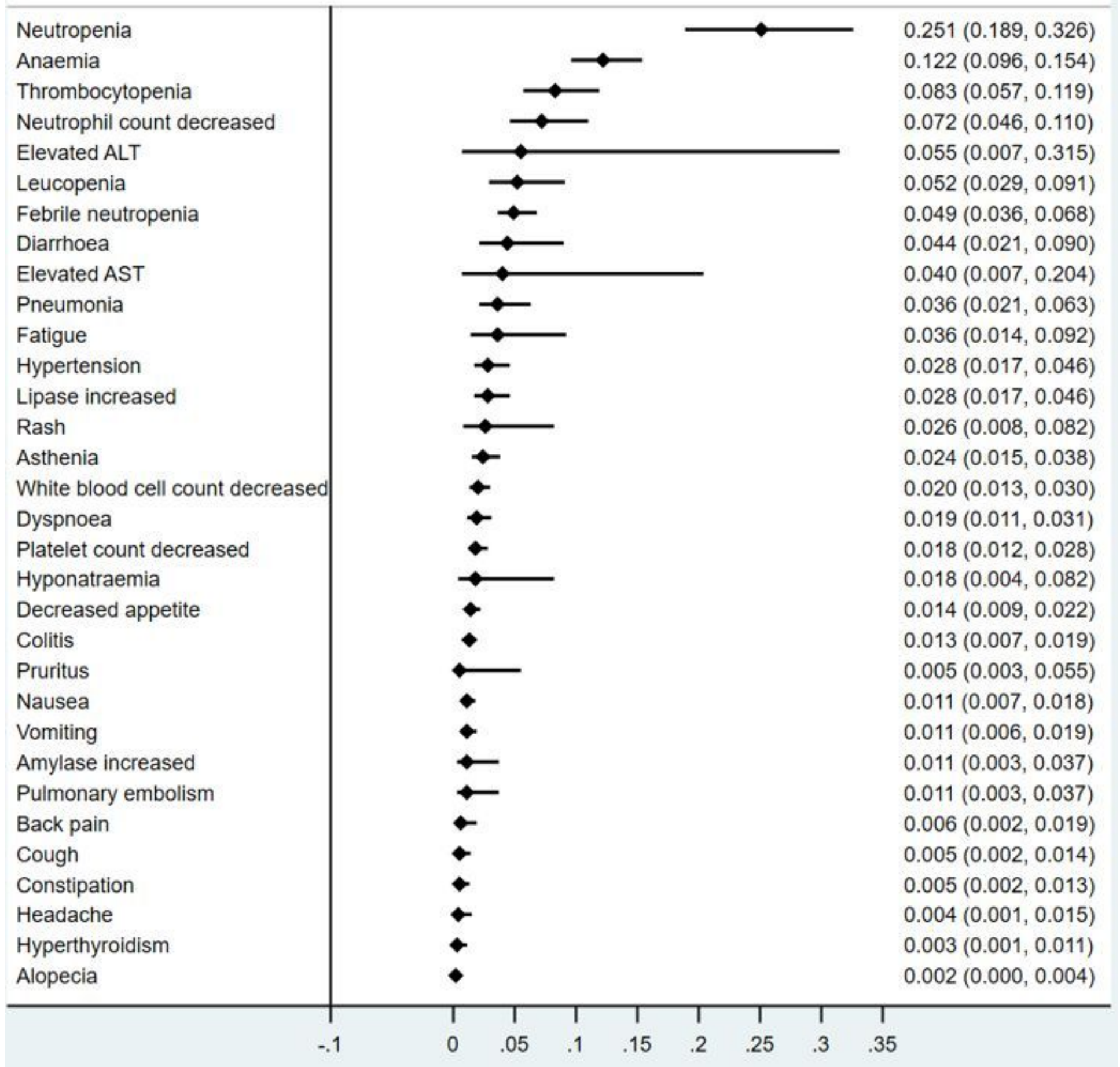

Figure 4

Rank forest plot of overall incidences of treatment-related adverse events in immunotherapy-based therapies.

\section{Supplementary Files}

This is a list of supplementary files associated with this preprint. Click to download. 
- supplementarymaterials.pdf 